\title{
Updated Statement on Endosurgical Training and Skills
}

\author{
Mohamed Mahmood Nasr, MB, BCh, MSC, FRCSI, MD, FIMSAT (Dundee, UK) ${ }^{1 *}$, Maged \\ Mahmoud Nasr, MB, BCh, MSc, MRCOGI ${ }^{2}$ Lamia Hamed Shehata ${ }^{3}, M B, B C h, M S C$ \\ ${ }^{1}$ Endoscopic \& General Surgeon, King Fahd Hospital, Riyadh, Ministry of Health, Kingdom of Saudi Arabia \\ ${ }^{2}$ Department of Obstetrics \& Gynecology, Endoscopic Surgeon, Obstetrician \& Gynecologist, Mazahmiya General \\ Hospital, Riyadh, Ministry of Health, Kingdom of Saudi Arabia \\ ${ }^{3}$ Department of Radiology, Diagnostic Radiologist, Care National Hospital, Riyadh, Kingdom of Saudi Arabia
}

\begin{abstract}
Objective: The surgical training for endoscopic proficiency program is a collaborative project between "Society of American Gastrointestinal and Endoscopic Surgeons" (SAGES) and Olympus America Inc. dedicated to providing flexible endoscopy Training to surgery residency programs. Currently it lacks models for proficiency-based training. A "Surgical Training for Endoscopic Proficiency" (STEP) Program has been suggested through several research projects. Recent developments in surgical technologies in general and machine learning in particular have transformed the way experts envision the future of surgery. Surgical technology is aiming to improve the quality of interventional healthcare. In this publication, we review current endoscopic surgery practice, essential professional skills, training practices and finally describe a roadmap for a standard professional training program specification that grants gaining the required skills.

Review and conclusions: The structured training framework should be the ideal model ensure safe implementation with development of metrics to grade successful learning and assessment of short- and long-term outcomes. The training improvement aspects should involve the following points:

The Accreditation Council for Graduate Medical Education, RESEARCH ADRESSED COMPETENCIES: According to the "The Accreditation Council for Graduate Medical Education (ACGME)", Second Revision (Version 2), [1] published on January 2019, this research deals with related professional surgical skills as demonstrated in (Table 1).

\section{Keywords}

Endo-Surgery Training, Cognitive, Meta-Cognitive and Spatial Cognitive Skills, Subjective and Objective Parameters, Automaticity, Visual Force Feedback Systems, Motor Imagery and Action Observation

\section{Abbreviations}

Society of American Gastrointestinal and Endoscopic Surgeons (SAGES); Surgical Training for Endoscopic Proficiency (STEP); Visual analogue scoring (VAS); Multiple choice questions (MCQs); Fundamentals of Endoscopic Surgery (FES); Motor imagery (MI); Action observation (AO); The National Aeronautics and Space Administration (NASA); Task Load Index (TLX); Visual-spatial secondary task (VSST); Objective assessment skills scales (OSATS); Motor imagery (MI)
\end{abstract}

\section{Methodology of Research}

\section{Search Strategy}

This systematic review is conducted in accordance with the "Preferred Reporting Items for Systematic Reviews and Meta Analyses" (PRISMA) guidelines. The Cochrane Library, Ovid, PubMed and Embase are searched for the following keywords: Endo-surgery training; Cognitive, metacognitive and spatial cognitive skills; Subjective and objective parameters; Automaticity; Visual force feedback systems; motor imagery and action observation.

Publications between January $1^{\text {st }}, 1990$ and December $1^{\text {st }}, 2020$ are included using search filters. In case of repeated
*Corresponding author: Dr. Mohamed Mahmood Nasr, MB, BCh, MSc, FRCSI, MD, FIMSAT (Dundee, UK) Endoscopic \& General Surgeon, King Fahd Hospital, Riyadh, Ministry of Health, Kingdom of Saudi Arabia, Tel: 966- 543-606-109.

Accepted: June 18, 2021

Published online: June 21, 2021

Citation: Mohamed MN, Maged MN Shehata LH (2021) Updated Statement on Endosurgical Training and Skills. Adv Laparoscopy $4(1): 87-92$ 
Table 1: Patient Care 3: Intra-Operative Patient Care - Technical Skills: Levels 3-5 [1].

\begin{tabular}{|l|l|l|l|}
\hline $\begin{array}{l}\text { Skill Level } \\
\text { Interpretation Stages }\end{array}$ & $\mathbf{L}$ & Level $\mathbf{3}$ & Level $\mathbf{5}$ \\
\hline STAGE 1 & $\begin{array}{l}\text { Consistently demonstrates careful } \\
\text { tissue handling. }\end{array}$ & $\begin{array}{l}\text { Adapts tissue handling based on } \\
\text { tissue quality. }\end{array}$ & Identifies innovative \\
\hline STAGE 2 & $\begin{array}{l}\text { Visualizes tissue plane, identifies } \\
\text { and dissects relevant normal } \\
\text { anatomy. }\end{array}$ & $\begin{array}{l}\text { Visualizes tissue plane, identifies } \\
\text { and dissects relevant abnormal } \\
\text { anatomy. }\end{array}$ & $\begin{array}{l}\text { Operative techniques, } \\
\text { instrument, operative approaches } \\
\text { and significant improvement in } \\
\text { established techniques }\end{array}$ \\
\hline STAGE 3 & $\begin{array}{l}\text { Moves fluidly through the course } \\
\text { of the operation and anticipates } \\
\text { next steps. }\end{array}$ & $\begin{array}{l}\text { Adapts the unexpected findings } \\
\text { and events during the course of } \\
\text { the operation. }\end{array}$ & \\
\hline
\end{tabular}

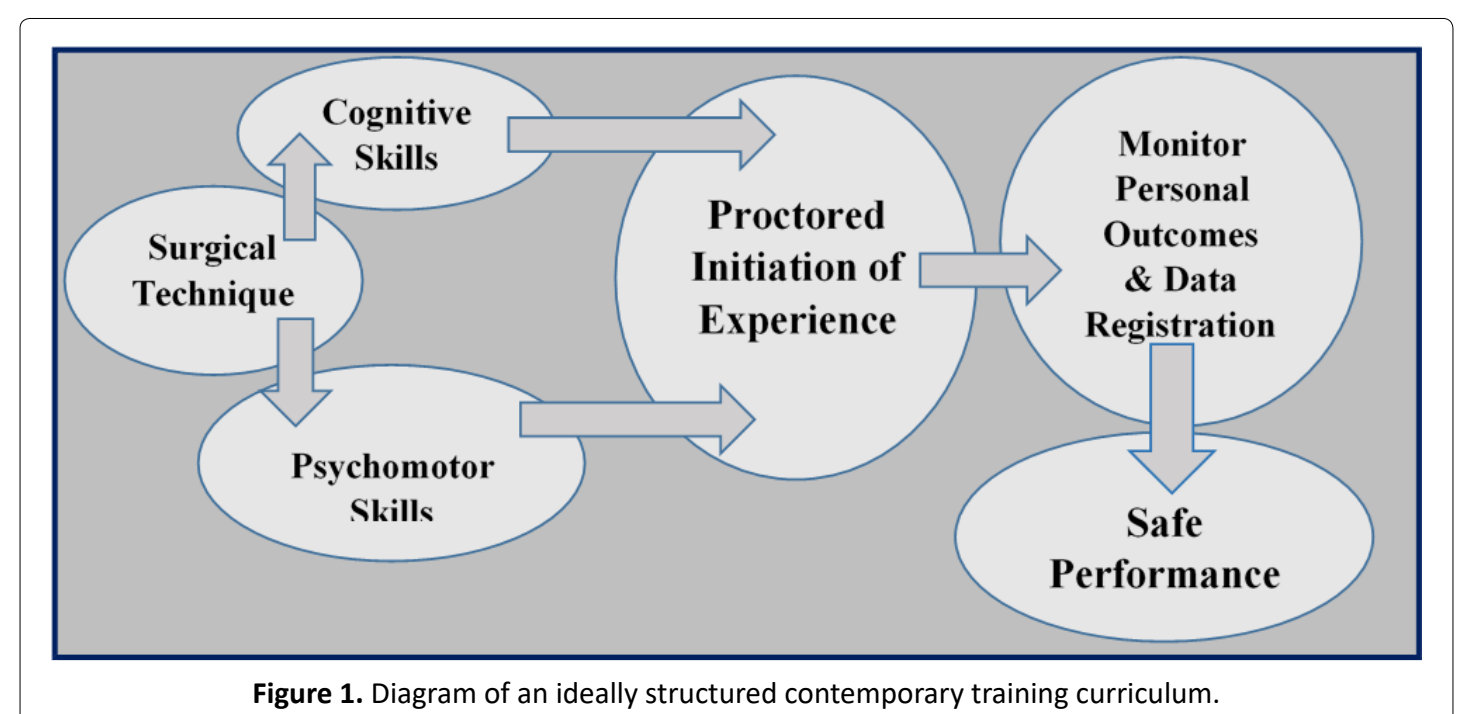

literature content, the most recent covering articles are included with exclusion of that of older date. Original articles and reviews are included with Cochrane Library, Ovid, PubMed and Embase filters.

\section{Literature Review}

All citations were managed in Microsoft Excel. Duplicate publications were removed. Inclusion criteria were as follows: initial publication date after January $1^{\text {st }}, 1990$ through December $1^{\text {st }}, 2020$; reporting on all related aspects to geriatric health care are all collected. Exclusion criteria included articles not available in English. All discrepancies were resolved through discussion among authors. The primary search has contained 746 full-length articles that were reviewed for inclusion in the systematic review. Study selection was unlimited to the type but relies on the high level of evidence and support of literature to the extracted information. Data extraction from reports achieved independently and confirming data with literature review for assurance of further evidence.

\section{Aim of Article}

The main aim of this article is to construct contemporary complete review on all aspects related the current situation of endo-surgical training and the proved and potential aspects necessary to modernize in relation to outcomes needed to gain, namely, standard endo-surgical skills, practice automaticity and skill-full professionalism through a fast-learning curve and efficient teaching procedure.

\section{Introduction}

The surgical training for endoscopic proficiency program is a collaborative project between "Society of American Gastrointestinal and Endoscopic Surgeons" (SAGES) and Olympus America Inc. dedicated to providing flexible endoscopy Training to surgery residency programs. Currently it lacks models for proficiency-based training. A "Surgical Training for Endoscopic Proficiency" (STEP) Program has been suggested through several research projects [2].

Recent developments in surgical technologies in general and machine learning in particular have transformed the way experts envision the future of surgery. Surgical technology is aiming to improve the quality of interventional healthcare. In this publication, we review current endoscopic surgery practice, essential professional skills, training practices and finally describe a roadmap for a standard professional training program specification that grants gaining the required skills. In the following exploitation, we will review the different items related to modernize endo-surgical training and finally conclude the suggested evidence-based training program. 


\section{Review of Literature}

\section{Value of endo-surgical skills courses}

The acquisition of endo-surgical skills represents an essential component and has been demonstrated that attending intensive short courses can improve both knowledge and skills in evidence-based medicine. Training on simulators recreates operative conditions and the skills acquired are effectively transferred to the operating room enhancing operative performance. However, curriculums vary considerably, and no consensus exists regarding the appropriate content or quality necessary for training. Upon evaluation of the effectiveness of 'hands-on' endo-surgical skills course through subjective assessment involved each attendee self-scoring their own knowledge in laparoscopy in different fields using "visual analogue scoring" (VAS), objective assessment through "multiple choice questions" (MCQs) and motor skill tasks and the difference between the performances of each of the candidates at pre- and postcourse periods was evaluated using the Wilcoxon signed rank test. There is dramatic improvement in the performing of motor skills in the dry laboratory. Such courses result in a short-term measurable improvement with recommendation to focus on assessing the impact of such 'hands-on' courses on the long term [3].

\section{Improving training of endo-surgical tissue manipulation skills using various visual force feedback types}

Visual force feedback allows trainees to learn endosurgical tissue manipulation skills. One study has recruited unexperienced medical students randomized three groups: "Constant Force Feedback", "Bandwidth Force Feedback" and "Fade-in Force Feedback". All participants performed a pretest, training, post-test and follow-up test. The study involved two dissimilar tissue manipulation tasks, one for training and one to assess transferability. All visual force feedback methods showed to be effective in decreasing tissue manipulation force as no major differences among groups in the post and follow-up trials. The "Bandwidth Force Feedback" method is preferred for respecting individual progress and minimizing distraction.

All visual force feedback groups showed to be equally effective in decreasing participants applied task force. The learning curves recorded in training, the mean scores of the force parameters in post-test and the retention effects a week after indicate that training with visual force feedback results in enhanced endo-surgical tissue manipulation skills. As the Bandwidth Force Feedback type is only present when force levels are dangerous, minimizing attentional distraction and so is preferable for training [4].

\section{Endo-surgical cognitive skills training}

Surgical competence is a multifactorial process and optimal training is based on acquiring knowledge and psychomotor and cognitive skills. Practicing surgical skills is one of the most crucial tasks for both the novice surgeon learning new procedures and surgeons already in practice updating techniques. Focus is placed on teaching traditional technical skills, but the importance of cognitive skills cannot be underestimated being allow to recognize environmental cues to improve technical performance including situational awareness, mental readiness, risk assessment, anticipating problems, decision-making, adaptation, and flexibility, in addition to accelerating the trainee's understanding of a procedure, formalize the steps being practiced, and reduce the overall training time to become technically proficient. There has been a paradigm shift in surgical education and consequently tools defining the steps for technical and cognitive learning have been developed. Framework structuring for ideal training models to ensure safe implementation has become a crucial target for professional bodies. Further development of metrics to grade successful learning and assessment of long term outcomes with the new pathway will confirm further success [5].

\section{Fundamentals of endo-surgical cognitive examination: Development and validity evidence}

The "Society of American Gastrointestinal and Endoscopic Surgeons" (SAGES) has developed the "Fundamentals of Endoscopic Surgery" (FES), serving to teach and assess the fundamental knowledge and skills required to practice flexible endoscopy of the gastrointestinal tract. The FES cognitive examination is the first test with validity evidence to assess the basic knowledge needed to perform flexible endoscopy. Combined with the hands-on skills examination, this assessment tool is a key component for FES certification due to the extensive validity evidence established with the goal of dissemination regardless of specialty. Continuity of this approach through adding updates is mandatory confirming success and competency in the field of endo-surgery [6].

\section{Cognitive assessment of endo-surgeons should include the following criteria:}

- Performance time is stable parameter for concentration and motor tests but visual test tends to be performed faster in cases with an intra-operative complication.

- Neither operative time nor unexpected intraoperative complication decreased surgeons' cognition.

- Surgeons showed great levels of resilience (defined as the extent to which individuals positively cope with work stress or adversity by adapting effectively, bouncing back from it, and maintaining or enhancing their well-being during adverse situations).

- Interestingly, resilience has become the theme of many presidential addresses from different international surgical societies; claimed as a desirable characteristic to excel in surgery. [7-10].

\section{Implementing cognitive training into endo- surgical skill course}

Minimally invasive surgery has challenged surgeons' skills through extending their usual haptic space and displaying indirect visual feedback through a screen, requiring new mental abilities, including spatial orientation and mental 
representation. In literature, studies have aimed testing the effect of cognitive training based on "motor imagery" (MI) and "action observation" (AO) on surgical skills. The most recent study has concluded two main results: first, $\mathrm{MI}$ associated with $\mathrm{AO}$ may be inefficient in learning new surgical skills when appropriate conditions of learning are not provided and phrase it more efficiently, it is assumed that $\mathrm{MI}$ and $\mathrm{AO}$ should be used as follows: First, cognitive training should not be implemented into a condensed training program; this would require much more time to assimilate additional mental workload brought by $\mathrm{Ml}$ and $\mathrm{AO}$; and second, cognitive training should be more adapted for intermediate to advanced surgeons to strengthen already acquired and memorized techniques (in particular, those related to spatial abilities). Training under cognitive load benefitted performance on an actual surgical task under similar conditions. In addition, completely self-directed training with the use of e-learning can be a feasible training approach to achieve technical proficiency in laparoscopic suturing and knot tying in a training setting. Collaboration between surgeons and cognitive neuroscientists specialized in motor learning is one of the main means to complete data and results that are already available and to add several upgrades to future investigations. To enable a learning curve, surgical performance should be evaluated on more trials and over a longer training period. Inter-rater and intra-rater reliability measures would provide more accuracy, and a demographic questionnaire about habits (e.g. use of virtual reality games) of the participants would complete the understanding of spatial abilities. [11-14].

\section{Significance of meta-cognitive skills in endo- surgery assessed by essential task simulation}

Metacognition is defined as the knowledge about one's own methods of perceiving, remembering, thinking, and acting. Metacognitive skills related to endo-surgery with the aim of applying the findings in endo-surgical training program. The following data is measured; longest performance times, longest path lengths, and most frequent tissue damage occurred at $135^{\circ}$ and at $180^{\circ}$ of the scope field of vision. There are significant correlation coefficients between the instrument path length and task difficulty (metacognition) at $135^{\circ}(\mathrm{R}=$ $0.74, p=0.03)$ and $180^{\circ}(R=0.79, p=0.02)$. The significance of metacognitive skills in endo-surgery is elucidated and so a training program should include recognition feedback systems to enrich the training outcomes [15].

\section{Subjective and objective quantification of the effect of distraction on surgeon's performance during simulated endo-surgery}

Distracting interference cognitive tasks place undeniable pressure on the minds of who need high precision and attention during the tasks, such as those performed during surgery; these tasks might affect ongoing surgical procedures. One study measured the effect of additional cognitive tasks on the mental load of the surgeon by measuring the mean change in pupil size, blink rate, and subjective assessment during surgery. The participants then performed the cognitive task (arithmetic problem), after that they performed the surgical task while completing the cognitive task on the simulator. All participants wore trackers to monitor pupil size and blink rate during surgery and the cognitive task. The "National Aeronautics and Space Administration" (NASA) "Task Load Index" (TLX) score also recorded performance parameters during the surgical mission. The double-task pupil size and the blink rate were significantly increased compared to the single-task observation, and the associated increase in psychological load might have been affected by surgical performance, and the performance parameters were also statistically significant. However, for the aforementioned parameters, experienced surgeons had some differences compared with inexperienced surgeons, but these differences did not reach statistical significance.

Distracted cognitive task stimulation in the operating room can increase the surgeon's psychological burden while also affecting their operational skills, thereby threatening patient safety; reduced cognitive costs might be obtained by improving or managing cognitive deficits [16].

\section{Spatial cognition through the keyhole}

Studying minimally invasive surgery can provide insights into basic processes of spatial cognition. Modeling these may provide benefits for this real-world domain. Evidently there are numerous situations in surgery that could benefit substantially from modeling approaches. If modeling can clarify some of the underlying processes it could be tremendously valuable to surgical educators and human factors researchers. Here after some specific questions for which modeling approaches might provide some traction.

- First question; studying medical education has shown that medical professionals acquire genuinely spatial mental models of anatomy that is subject to individual differences in spatial abilities. An important future goal will be to characterize how individuals learn these spatial concepts and to develop educational approaches ameliorating individual differences.

- Second question; the relationship between performance and ability is dynamic; changing over time as experience is acquired. The changing role of general cognitive abilities and what happens to spatial cognitive abilities in spatially demanding domains is still not so clear demanding future research to clarify how and why different abilities shift in importance over time.

- Third question; is whether initial personal differences alter through or even disappear completely at the end of training, and whether amount and type of training depends on the starting abilities of the individual. This depends on task-specific factors as some spatial tasks may be learnable by everyone with enough training, whereas others may not. Alternatively, it is possible that individual differences, specifically the influence of spatial abilities, never disappears completely. Collectively, empirical data and principled theoretical model-building of the underlying changes that occur over time are needed to reach a conclusion on these very important issues [17]. 
Individual differences seem to play an important, and dynamic, role in spatial domains such as this one. A key question is whether variance in spatial ability is due to qualitative differences (e.g., recruitment of different mechanisms or engagement of different strategies) or quantitative differences (e.g. capacity limitations or speed of processing differences within universal underlying architectures). Thomas and Karmiloff-Smith (2003) optimistically note that, compared to the complexities of studying domain-general cognition, modeling the variation in domain-specific processes such as spatial or verbal functions is probably quite tractable. Continued progress through the modeling community in this regard; making a genuine contribution to skilled performance and learning in this societally important real-world domain [18].

\section{Validation of a visual-spatial secondary task to assess automaticity related to endo-surgery skills}

Assessment of reliability and validity of a "visual-spatial secondary task" (VSST) as a method to measure automaticity on a basic simulated endo-surgical skill model holds crucial importance that is for motor skill acquisition, expertise is defined by automaticity. The highest level of performance with less cognitive and attentional resources characterizes this stage, allowing expert stopper form multiple tasks.

Conventional validated parameters as operative time, "objective assessment skills scales" (OSATS), and movement economy, are insufficient to distinguish if an individual has reached the more advanced learning phases, such as automaticity. There is literature about using a VSST as an attention indicator correlating with the automaticity level. Novices with completed and approved fundamentals of endosurgery course, and endo-surgery experts were enrolled for an experimental study and measured under dual tasks conditions. Each participant performed the test giving priority to the primary task while at the same time they responded to a VSST. The primary task consisted of four interrupted laparoscopic stitches (ILS) on a bench-model. The VSST was a screen that showed different patterns that the surgeon had to recognize and press a pedal while doing the stitches (Psycho Psysoftware, Python, MacOS). Novices were over trained on ILS until they reach at least 100 repetitions and then were retested.

Participants were video recorded and then assessed by 2 blinded evaluators whom assured operative time and OSATS. These scores were considered indicators of quality for the primary task. The VSST performance was measured by the detectability index (DI), which is the ratio between correct and wrong detections. A reliable evaluation was defined as two measures of DI with less than $10 \%$ of difference, maintaining the cut off scores for performance on the primary task (operative time $<110$ seconds and OSATS $>17$ points).

By categorizing DI based on expert performance, novices with DI-post 40.65 achieved better OSATS score and less operative time than novices with DI-posto 0.65 ( $p=0.007 \mathrm{y}$, $p=0.089$, respectively). Measuring automaticity is feasible using a VSST. This instrument is reliable and has a face, content and construct validity. DI value over 0.65 may be a cut-off point correlated with high standard performance on the primary task. This instrument measures performance on endo-surgical skills, and along with conventional indicators, would better define advance levels of expertise [19].

\section{Conclusions}

\section{Suggested endo-surgical training methodology}

The suggested framework should be the ideal model for structured training to ensure safe implementation with development of metrics to grade successful learning and assessment of short- and long-term outcomes. This framework would act on "The Accreditation Council for Graduate Medical Education (ACGME)"; surgical skills related to intra-operative patient care - technical skills through levels 3-5.

The following points are suggested for structuring a standard program as extracted from literature review:

1. Endo-surgeons selection should involve cognitive assessment that include procedural performance time being a stable indicator for concentration and motor tests, visual test that tends to be performed faster in cases with an intra-operative complication and finally surgeon's resilience.

2. The significance of metacognitive skills in endo-surgery is elucidated dictating that a training program should include recognition feedback systems.

3. Subjective and objective parameters are significant monitors for surgeon's professional performance.

4. Automaticity measurement is a training cut-off point correlated to high standard performance on the primary task is of extreme significance mandating its application.

5. All visual force feedback systems ["Constant Force Feedback", "Bandwidth Force Feedback" and "Fadein Force Feedback"] showed to be equally effective in decreasing participants applied task force. The learning curves recorded in training, the mean scores of the force parameters in post-test and the retention effects after a week indicate that training with visual force feedback results in enhanced endo-surgical tissue manipulation skills. As the Bandwidth Force Feedback type is only present when force levels are dangerous, it minimizes attentional distraction and is therefore the preferable system for training.

6. Cognitive training based on "motor imagery" (MI) and "action observation" (AO) should be applied in endosurgery training as follows; first, cognitive training should not be implemented into a condensed training program; acquiring much more time to assimilate additional mental workload brought by $\mathrm{MI}$ and $\mathrm{AO}$; and second, cognitive training should be more adapted for intermediate to advanced surgeons to strengthen the already acquired and memorized techniques. Involvement of cognitive neuroscientists specialized in motor learning is one of the main means to implement efficient training programs leading to fast-surging learning curves.

7. Training modelling through spatial cognitive insights positively enhances the outcome.

\section{Declaration statements}

Authors' contributions: All authors whose names appear on the submission have made equal contributions to all of the following: 
a) The conception and design of the study, acquisition of data and interpretation of data,

b) Drafting the article and revising it critically for important intellectual content,

c) Final approval of the submitted research version,

d) Agree to be accountable for all aspects of the work in ensuring that questions related to the accuracy or integrity of any part of the work are appropriately investigated and resolved.

Conflicts of interest / Competing interests: All authors declare nothing to disclose regarding the followings: corporate/commercial relationships including all consultantships, honoraria, stock ownership, gifts, free or reimbursed travel / vacations, equity interests, arrangements regarding patents or other vested interests, that might pose a conflict of interest.

Funding: All authors declare nothing to disclose related to any aspect of funding.

Availability of data and material (data transparency): Data sharing and data citation aspects are transparently achieved.

Consent to participate \& publication: Inapplicable.

Code availability: In Applicable.

Ethical approval: Inapplicable.

\section{Highlights}

- Endo-surgeons selection should involve cognitive assessment; being crucial in gaining inter-active training motor skills as documented in the points below:

1. The metacognitive skills in endo-surgery.

2. Subjective and objective parameters as significant monitors for surgeon's professional performance.

3. Automaticity measurement being a training cut-off point correlated to high standard performance.

4. All visual force feedback systems "Constant Force Feedback", "Bandwidth Force Feedback" and "Fade-in Force Feedback" being equally effective in decreasing participants applied task force.

5. Cognitive training based on "motor imagery" and "action observation" should be applied in endo-surgery training in consequence.

6. Spatial cognitive insights positively enhance the training outcome.

\section{References}

1. "The Accreditation Council for Graduate Medical Education (ACGME)"; (2019) Second Revision (Version 2).

2. Wilcox V, Trus T, Salas N, et al. (2014) A proficiency-based skills training curriculum for the SAGES Surgical Training for Endoscopic Proficiency (STEP) program. J Surg Educ 71: 282-288.
3. Condous G, Alhamdan D, Bignardi $T$, et al. The value of laparoscopic skills courses Aust N Z J Obstet Gynaecol 49: 312-315.

4. Smit D, Spruit E, Dankelman J, et al. (2017) Improving training of laparoscopic tissue manipulation skills using various visual force feedback types. Surg Endosc 31: 299-308.

5. Knol J, Keller DS (2019) Cognitive skills training in digital era: A paradigm shift in surgical education using the TaTME model. Surgeon 17: 28-32.

6. Poulose B, Vassiliou M, Dunkin B, et al. (2014) Fundamentals of endoscopic surgery cognitive examination: development and validity evidence. Surg Endosc 28: 631-638.

7. Beneduzzi J, Herbella F, Schlottmann F, et al. (2019) Cognitive assessment of surgeons during surgical procedures: Influence of time and intraoperative complications. World J Surg 43:143-148.

8. Vickers S, Vickers A (2017) Lessons learned from mentors and heroes on leadership and surgical resilience. J Gastrointest Surg 21: 1-11.

9. Kurek S Jr. (2016) EAST 2016 presidential address: Resilience. J Trauma Acute Care Surg 81:1-7.

10. Minter R (2018) Passion, integrity, resilience: Core values to carry us forward 2017 Society of University Surgeons presidential address. Surgery 163: 996-1001.

11. De Witte B, Di Rienzo F, Martin X, (2018) Implementing cognitive training into a surgical skill course: A pilot study on laparoscopic suturing and knot tying. Surgical Innov 25: 625-635.

12. Schmidt M, Kowalewski K, Trent S, et al. (2020) Self-directed training with e-learning using the first-person perspective for laparoscopic suturing and knot tying: a randomised controlled trial: Learning from the surgeon's real perspective. Surg Endosc. 34: 869-879.

13. Sankaranarayanan G, Odlozil C, Wells K, et al. (2020) Training with cognitive load improves performance under similar conditions in a real surgical task. Am J Surg 220: 620-629.

14. Ritter F, Yeh M, Yan Y, et al. (2020) Effects of varied surgical simulation training schedules on motor-skill acquisition. Surg Innov 27: 68-80.

15. Uemura M, Tomikawa M, Nagao $Y$, et al. (2014) Significance of metacognitive skills in laparoscopic surgery assessed by essential task simulation. Minimally Invasive Ther Allied Technol 23: $165-172$.

16. Gao J, Liu S, Feng Q, et al. (2019) Subjective and objective quantification of the effect of distraction on physician's workload and performance during simulated laparoscopic surgery. Med Sci Monit 25: 3127-3132.

17. Keehner M (2011) Spatial cognition through the keyhole: How studying a real-world domain can inform basic science - and vice versa. Top Cogn Sci 3: 632-647.

18. Thomas M, Karmiloff-Smith A (2003) Connectionist models of development, developmental disorders and individual differences. In R J Sternberg, J Lautrey, T Lubart (Edn), Models of intelligence: International perspectives, American Psychological Association, Washington, DC, USA 133-150.

19. Castillo R, Alvarado J, Moreno P, et al. (2017) Validation of a visual-spatial secondary task to assess automaticity in laparoscopic skills. J Surg Educ 75: 1001-1005. 